\title{
The effects of the guide field on the structures of electron density depletions in collisionless magnetic reconnection
}

\author{
LU San ${ }^{1,2}$, LU QuanMing $^{1 *}$, CAO Yang ${ }^{3}$, HUANG Can ${ }^{1}$, XIE JinLin ${ }^{3} \&$ WANG Shui ${ }^{1}$ \\ ${ }^{1}$ CAS Key Laboratory of Basic Plasma Physics, School of Earth and Space Sciences, University of Science and Technology of China, Hefei \\ 230026, China: \\ ${ }^{2}$ State Key Laboratory of Space Weather, Chinese Academy of Sciences, Beijing 100190, China; \\ ${ }^{3}$ CAS Key Laboratory of Basic Plasma Physics, School of Physics, University of Science and Technology of China, Hefei 230026, China
}

Received October 8, 2010; accepted October 17, 2010

\begin{abstract}
Two-dimensional particle-in-cell simulations are performed to investigate the formation of electron density depletions in collisionless magnetic reconnection. In anti-parallel reconnection, the quadrupole structures of the out-of-plane magnetic field are formed, and four symmetric electron density depletion layers can be found along the separatrices due to the effects of magetic mirror. With the increase of the initial guide field, the symmetry of both the out-of-plane magnetic field and electron density depletion layers is distorted. When the initial guide field is sufficiently large, the electron density depletion layers along the lower left and upper right separatrices disappear. The parallel electric field in guide field reconnection is found to play an important role in forming such structures of the electron density depletion layers. The structures of the out-of-plane magnetic field $B_{y}$ and electron depletion layers in anti-parallel and guide field reconnection are found to be related to electron flow or in-plane currents in the separatrix regions. In anti-parallel reconnection, electrons flow towards the $X$ line along the separatrices, and are directed away from the $X$ line along the magnetic field lines just inside the separatrices. In guide field reconnection, electrons can only flow towards the $X$ line along the upper left and lower right separatrices due to the existence of the parallel electric field in these regions.
\end{abstract}

magnetic reconnection, electron density depletion, quadrupole structures, separatrices, in-plane current

Citation: $\quad$ Lu S, Lu Q M, Cao Y, et al. The effects of the guide field on the structures of electron density depletions in collisionless magnetic reconnection. Chinese Sci Bull, 2011, 56: 48-52, doi: 10.1007/s11434-010-4250-9

Magnetic reconnection is thought to be one of the most important mechanisms that converts rapidly magnetic energy into kinetic energy of plasma. At the same time, the topological configuration of the magnetic field changes. Magnetic reconnection plays an important role in space and laboratory plasma as a driving mechanism for many explosive phenomena, such as solar flares, substorms in the Earth's magnetosphere and disruptions in laboratory fusion experiments [1-5].

At scale lengths greater than the ion inertial length, $c / \omega_{\mathrm{pi}}$, magnetohydrodynamic theory is valid, because ions and electrons are both frozen in the magnetic field lines. However, if we study magnetic reconnection at scale lengths between the ion inertial length and electron inertial length

*Corresponding author (email: qmlu@ustc.edu.cn) (where the electron inertial length is $c / \omega_{\mathrm{pe}}$ ), only the electrons are frozen in the magnetic field lines, and ions can move across the magnetic field lines. This ion-electron decoupling causes Hall effects which are very important in collisionless magnetic reconnection [6-8]. At scale lengths below $c / \omega_{\text {pe }}$, the frozen-in constraint of the electrons is also broken, both ions and electrons can move across the magnetic field lines [7,9]. Sonnerup [6] proposed that the Hall effects can lead to the in-plane Hall current system, and the relations between the Hall current and the out-of-plane magnetic field have recently been studied extensively in anti-parallel reconnection. This can be roughly summarized as follows: Because of the magnetic mirror effect, electrons will flow toward the $X$ line along the separatrices; after accelerated in the vicinity of the $X$ line, the electrons flow 
away from the $X$ line along the magnetic field lines on the inner side of the separatrices [10,11]. Therefore, the in-plane Hall currents are directed away from the $X$ line along the separatrices, and toward the $X$ line just inside the separatrices [11-13]. A quadrupole structure of the out-ofplane Hall magnetic field with peaks between the regions carrying the in-plane currents is formed by such a current system [6,11-13]. At the same time, simulations and observation have shown that the electrons flow toward the $X$ line can form electron density depletion layers along the separatrices [11,14-16]. Therefore, the electron density depletion layers are outside the peaks of the out-of-plane magnetic field. This has already been observed during magnetic reconnection by Cluster satellites [11,12].

Recently, the guide field was found to have a significant impact on the structures of the out-of-plane magnetic field, whose symmetry is distorted $[17,18]$. In this paper, based on a two-dimensional (2D) particle-in-cell (PIC) simulation code, we explore the effects of the guide field on the structures of the electron density depletion layers. The relations among the in-plane Hall current, the electron density depletion layers, and the out-of-plane magnetic field, are also investigated.

\section{Simulation model}

In this paper, 2D PIC simulations are employed to investigate the structures of the electron density depletion layers in collisionless magnetic reconnection. In our simulation model, the electromagnetic fields are defined on the grids and updated by solving Maxwell equations with a full explicit algorithm. The ions and electrons are advanced in the electromagnetic fields [17]. The initial equilibrium configuration we adopt in this paper is a Harris current sheet model [19]. Therefore, the initial equilibrium magnetic field and density are given by

$$
\begin{gathered}
\boldsymbol{B}_{0}(z)=B_{0} \tanh \left[\left(z-\frac{L_{z}}{2}\right) / \delta\right] \boldsymbol{e}_{x}-B_{y 0} \boldsymbol{e}_{y}, \\
n(z)=n_{\mathrm{b}}+n_{0} \operatorname{sech}^{2}\left[\left(z-\frac{L_{z}}{2}\right) / \delta\right],
\end{gathered}
$$

where $B_{y 0}$ is the initial guide field, $L_{z}$ is the length of the domain in the $z$ direction, $\delta$ is the half-width of the current sheet, and $n_{\mathrm{b}}$ is the density of the background plasma. We adopt a Maxwellian distribution for each species of particles. The drift speeds in the $y$ direction satisfy $V_{\mathrm{i} 0} / V_{\mathrm{e} 0}=$ $-T_{\mathrm{i} 0} / T_{\mathrm{e} 0}$, where $V_{\mathrm{i} 0}\left(V_{\mathrm{e} 0}\right)$ and $T_{\mathrm{i} 0}\left(T_{\mathrm{e} 0}\right)$ are the drift speed and initial temperature of the ions (electrons), respectively. In our simulation, $T_{\mathrm{i} 0} / T_{\mathrm{e} 0}=4$, and $n_{\mathrm{b}}=0.2 n_{0}$. The half-width of the current sheet is $\delta=0.5 c / \omega_{\mathrm{pi}}$, where $c / \omega_{\mathrm{pi}}$ is the ion inertial length defined by the peak Harris density $n_{0}$. The mass ratio is set to be $m_{\mathrm{i}} / m_{e}=100$. The velocity of light is set to be $c=15$
$V_{\mathrm{A}}$, where $V_{\mathrm{A}}$ is the Alfven speed based on $B_{0}$ and $n_{0}$. The computation is carried out in a rectangular domain in the $x-z$ plane. The number of simulation grids is $N_{x} \times N_{z}=256 \times 128$. The spatial resolution is $\Delta x=\Delta z=0.06 c / \omega_{\mathrm{pi}}=0.6 c / \omega_{\mathrm{pe}} \approx 2 \lambda_{D e}$ where $\lambda_{\mathrm{De}}$ is the electron Debye length. Therefore, the computation domain size is $L_{x} \times L_{z}=\left(15.36 c / \omega_{\mathrm{pi}}\right) \times\left(7.68 \mathrm{c} / \omega_{\mathrm{pi}}\right)$. The time step is $\Omega_{\mathrm{i}} t=0.0001$, where $\Omega_{\mathrm{i}}$ is the ion gyro-frequency. In all runs we employ more than a million particles per species to represent the plasma of the Harris current sheet, and the same number of particles is used to represent the background plasma. The periodic boundary conditions are used along the $x$ direction. At the same time, ideal conducting boundary conditions for the electromagnetic fields are used in the $z$ direction. Particles will be reflected when they reach the boundaries. The initial current sheet is modified, as in the Geospace Environmental Modeling Challenge reconnection problem, by including an initial flux perturbation [7].

\section{Simulation results}

2D PIC simulations are performed to investigate the effects of the initial guide on electron density depletion layers and Hall current system in collisionless magnetic reconnection. Four cases are simulated with different initial guide fields $B_{z 0} / B_{0}=0,0.2,0.6$ and 1 .

Figure 1 shows the time evolution of the reconnected magnetic flux $\Delta \psi$ for cases $B_{y 0} / B_{0}=0, B_{y 0} / B_{0}=0.2, B_{y 0} / B_{0}=$ 0.6 , and $B_{y 0} / B_{0}=1$. Here the magnetic flux $\Delta \psi$ is defined as the flux difference between the $X$ and $O$ lines, and its slope can be served as an indicator of the magnetic reconnection rate. Similar to previous simulations, the reconnection rate is found to decreases substantially when the guide field is sufficiently large $\left(B_{y 0} / B_{0} \sim 0.5\right)$.

Figure 2 shows contours of the out-of-plane magnetic fields $B_{y}^{\prime} / B_{0}=\left(B_{y}+B_{y 0}\right) / B_{0}$ for cases (a) $B_{y 0} / B_{0}=0$ at $\Omega_{0 i} t=18$, (b) $B_{y 0} / B_{0}=0.2$ at $\Omega_{0 i} t=19$, (c) $B_{y 0} / B_{0}=0.6$ at $\Omega_{0 i} t=19$ and (d) $B_{y 0} / B_{0}=1$ at $\Omega_{0 i} t=23$. In the figure, the out-of-plane magnetic field is chosen at the time when the maximum reconnection

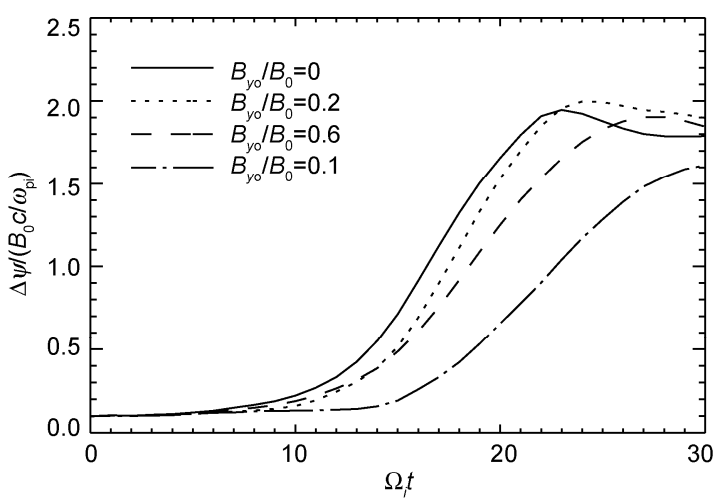

Figure 1 The time evolution of the reconnected magnetic flux $\Delta \psi$ for cases $B_{y 0} / B_{0}=0,0.2,0.6$ and 1.0 . 

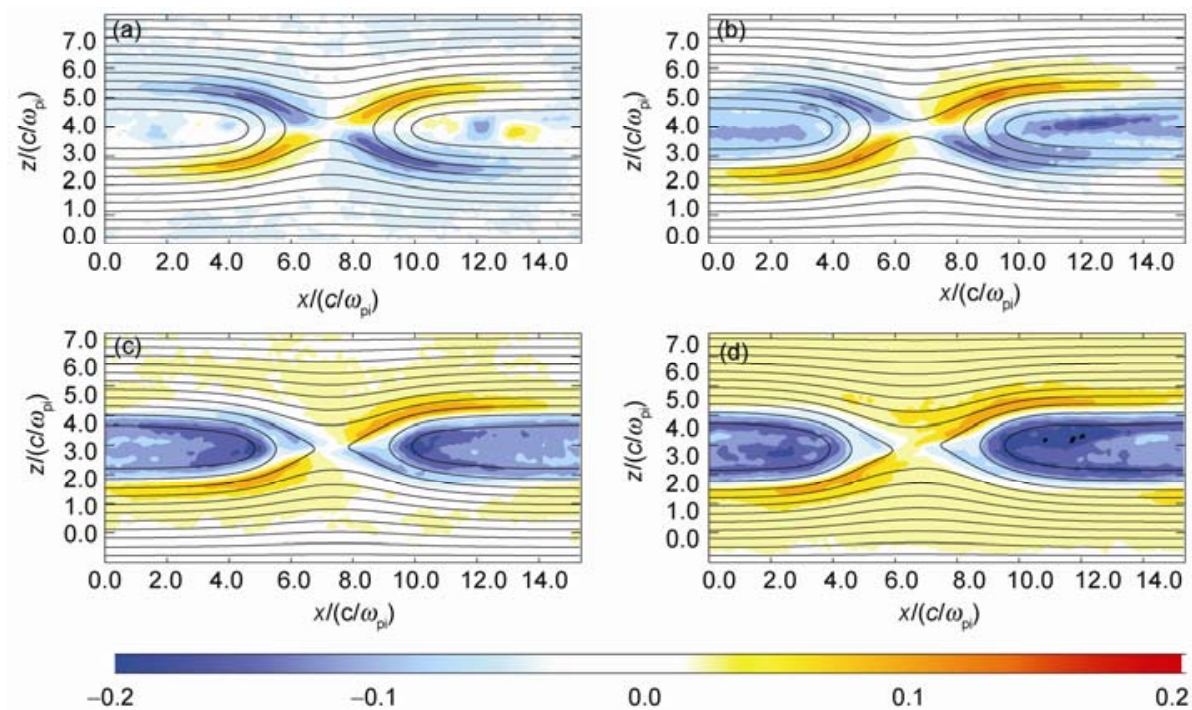

Figure 2 Contours of the out-of-plane magnetic fields $B_{\mathrm{y}}^{\prime} / B_{0}=\left(B_{y}+B_{y 0}\right) / B_{0}$ for cases (a) $B_{y} / B_{0}=0$ at $\Omega_{0 i} t=18$, (b) $B_{y} / B_{0}=0.2$ at $\Omega_{0 i} t=19$, (c) $B_{y 0} / B_{0}=0.6$ at $\Omega_{0 i} t=19$ and (d) $B_{y 0} / B_{0}=1$ at $\Omega_{0 i} t=23$. The corresponding magnetic field lines are also plotted in each figure.

rate is attained, and the magnetic field lines are also plotted. Obviously, in the case with $B_{y 0} / B_{0}=0$, the out-of-plane magnetic field $B_{y}^{\prime}$ forms a quadrupole structure with a maximum amplitude of about $0.1 B_{0}$. With the increase of the initial guide field, the negative parts of the quadrupole structures gradually move into the magnetic island and occupy most of the area inside it. Therefore, the quadrupole structures of $B_{y}^{\prime}$ are distorted. This phenomenon has been observed in numerous numerical studies $[17,18]$.

The structures of the electron density depletion layers can be found in Figure 3, which describe the contours of the electron density $n_{\mathrm{e}}$ for cases (a) $B_{y 0} / B_{0}=0$ at $\Omega_{0 i} t=18$, (b) $B_{y 0} / B_{0}=0.2$ at $\Omega_{0 i} t=19$, (c) $B_{y 0} / B_{0}=0.6$ at $\Omega_{0 i} t=19$ and (d) $B_{y 0} / B_{0}=1$ at $\Omega_{0 i} t=23$. In the case with $B_{y 0} / B_{0}=0$, electron density depletion layers can be clearly seen along the separatrices. With the increase of the initial guide field, the symmetry of the electron density depletion layers is distorted. When the initial guide field is sufficiently large $\left(B_{y 0} / B_{0} \sim 0.5\right)$, only the depletion layers along the separatrix from the upper left to the lower right exist.

The different structures of the out-of-plane magnetic field and electron density depletion layers in anti-parallel and guide field reconnection can be explained based on the electron flow or in-plane Hall currents. In Figure 4, electron and ion flow vectors in the $x-z$ plane are shown for cases (a) $B_{y 0} / B_{0}=0$ at $\Omega_{0 i} t=18$ and (b) $B_{y 0} / B_{0}=1$ at $\Omega_{0 i} t=23$. Because the ions are unmagnetized in the ion diffusion region, their flow speed is much smaller than that of the electrons.
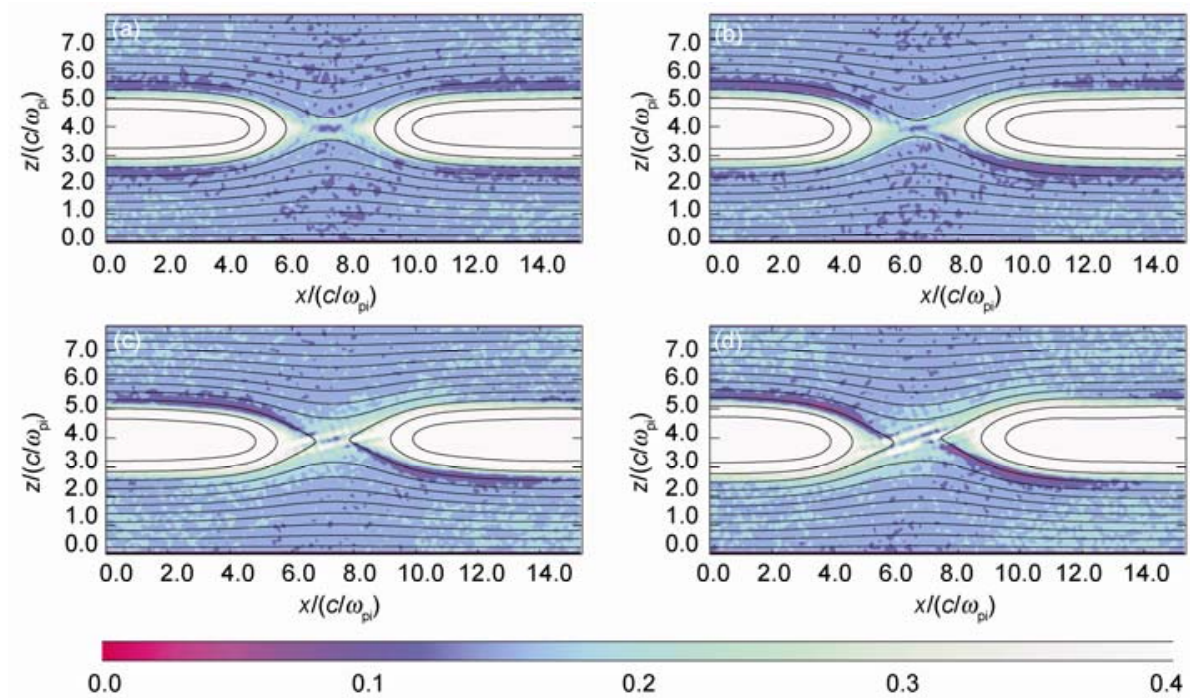

Figure 3 Contours of the electron density $n_{e}$ for cases (a) $B_{y 0} / B_{0}=0$ at $\Omega_{0 i} t=18$, (b) $B_{y 0} / B_{0}=0.2$ at $\Omega_{0 i} t=19$, (c) $B_{y 0} / B_{0}=0.6$ at $\Omega_{0 i} t=19$ and (d) $B_{y 0} / B_{0}=1$ at $\Omega_{0 i} t=23$. The corresponding magnetic field lines are also plotted in each figure. 
(a) $V_{e} \max =2.0 V_{A}$
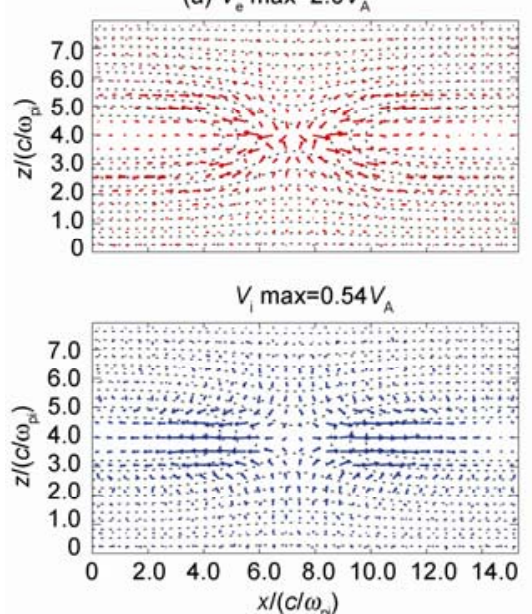

(b) $V_{e} \max =1.76 V_{A}$

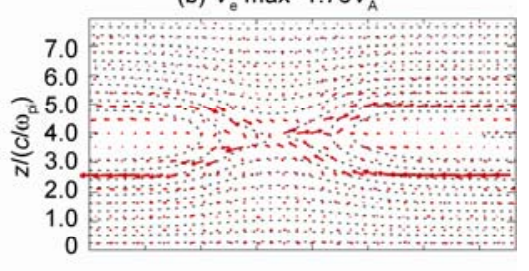

$V_{i} \max =0.52 V_{A}$

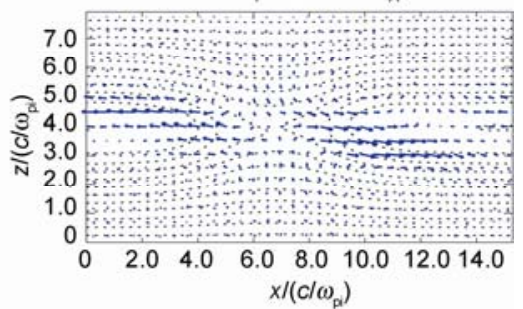

Figure 4 The electron and ion flow vectors in the $x-z$ plane for cases (a) $B_{y 0} / B_{0}=0$ at $\Omega_{0 i} t=18$ and (b) $B_{y 0} / B_{0}=1$ at $\Omega_{0 i} t=23$. The dashed lines denote the corresponding magnetic field lines.

Therefore, the contributions of ions to the in-plane Hall current are negligible. In anti-parallel reconnection, electrons flow toward the $X$ line along the separatrices due to the magnetic mirror, and then electron density depletion layers are formed along the separatrices [11]. After they are accelerated in the vicinity of the $X$ line, these electrons flow away from the $X$ line along the magnetic field lines just inside of the separatrices. In guide field reconnection, we can only find electrons flow toward the $X$ line along the separatrix from the upper left to the lower right, and high speed electrons flow away from the $X$ line inside the separatrix from the lower left to the upper right. In guide field reconnection, electrons are accelerated by the parallel electric field in the vicinity of the $X$ line, and when they move toward the $X$ line. This can be seen in Figure 5 , which shows the parallel electric field $\boldsymbol{E} \cdot \boldsymbol{B} / \boldsymbol{B}$ for case $B_{y 0} / B_{0}=1$ at $\Omega_{0 i} t=23$. The parallel electric field only exists along the separatrix from the upper left to the lower right, which forms the electron density depletion layers in these regions. The parallel electric field is caused by the gradient of the electron pressure. After these electrons are accelerated by the parallel electric field, they flow away from the $X$ line along the magnetic field lines just inside the separatrix from the lower left to the upper right.

The in-plane Hall current system is mainly determined by the electron flow, which is shown in Figure 4. According to the electron flow, we can calculate the in-plane Hall current along the magnetic field lines, which is shown in Figure 6. In anti-parallel reconnection, the currents are directed away from the $X$ line along the separatrices, and toward the $X$ line just inside the separatrices. Such an in-plane Hall current system leads to the quadrupole structures of the out-of-plane magnetic field. In guide field reconnection, the currents away from the $X$ line only exist along the separatrix from the upper left to the lower right, while the currents toward the $X$ line only exist inside the separatrix from the lower left to the upper right. Such an in-plane Hall current

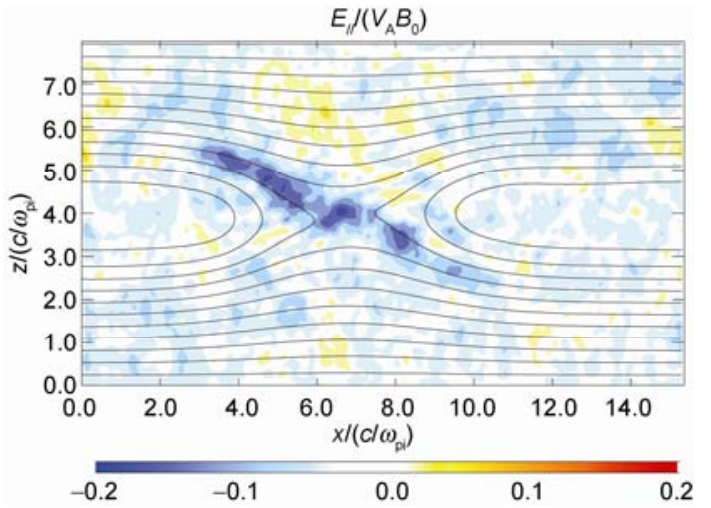

Figure 5 The parallel electric field $\boldsymbol{E} \cdot \boldsymbol{B} / \boldsymbol{B}$ for the case $B_{y_{0}} / B_{0}=1$ at $\Omega_{0 i} t$ $=23$. The corresponding magnetic field lines are also plotted in the figure.

system leads to the out-of-plane magnetic field $B y / B_{0}=$ $\left(B_{y}+B_{y 0}\right) / B_{0}$ that exists inside the magnetic island.

\section{Conclusions and discussion}

The effects of the guide field on the structures of the out-ofplane magnetic field and electron density depletion layer are investigated in this paper with the help of 2D PIC simulations. In anti-parallel reconnection, the electrons move toward the $X$ line along the separatrices because of the effects of the magnetic mirror, and they are directed away from the $X$ line along the magnetic field lines just inside the separatrices. Therefore, the in-plane Hall currents are directed away from the $X$ line along the separatrices, and toward the $X$ line just inside the separatrices. Such a current system leads to the quadrupole structures of the out-of-plane magnetic field $B_{y}$, and four symmetrical electron density depletion layers that are formed along the separatrices [11]. With the increase of the initial guide field, the symmetry of both $B_{y}$ and the electron density depletion layers are distorted. When the 


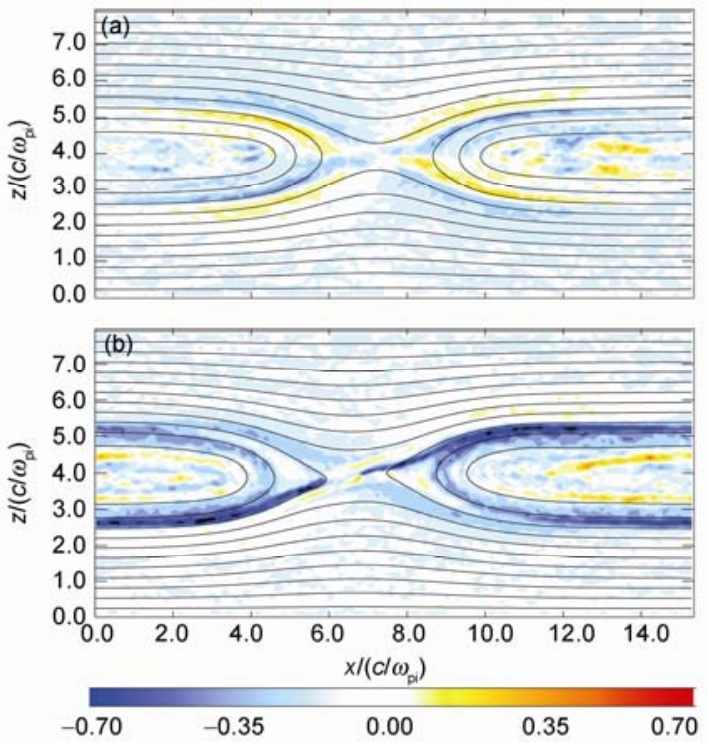

Figure 6 The in-plane Hall current along the magnetic field lines for cases (a) $B_{y 0} / B_{0}=0$ at $\Omega_{0 i} t=18$ and (b) $B_{y 0} / B_{0}=1$ at $\Omega_{0 i} t=23$. The corresponding magnetic field lines are also plotted in each figure.

initial guide field is sufficiently large, the parallel electric field in guide field reconnection, which is generated by the gradient of the electron pressure, is found to play an important role to form such structures. The parallel electric field only exists along the separatrix from the upper left to the lower right [20]. Electrons are accelerated by the parallel electric field in the vicinity of the $X$ line, as well as when they move toward the $X$ line along the separatrix from the upper left to the lower right. This forms the electron density depletion layer in this region. After these electrons are accelerated by the parallel electric field, they flow away from the $X$ line inside the separatrix from the lower left to the upper right. Correspondingly, the currents away from the $X$ line only exists along the separatrx from the upper left to the lower right, while the currents toward the $X$ line only exists inside the separatrix from the lower left to the upper right. We conclude that such an in-plane Hall current system lead to the out-of-plane magnetic field $B_{y}^{\prime} / B_{0}$ that exists inside the magnetic island.

Recently, the relations between the out-of-plane magnetic field and electron density depletion layers have been studied extensively in anti-parallel reconnection $[11,12]$. Electrons flow toward the $X$-line along the separatrices, and then are directed away from the $X$ line in the inner side of the separatrices. The out-of-plane Hall magnetic field forms a quadrupole structure with peaks between the regions carrying the in-plane currents. At the same time, the electrons flowing toward the $X$ line can form electron density depletion layers along the separatrices. Therefore, the electron density depletion layers are outside the peaks of the out-ofplane magnetic field. This has also already been observed during magnetic reconnection by Cluster satellites [11]. The situation changes when the guide field is sufficiently large. In guide field reconnection, the electron density depletion layers along the lower left and upper right separatrices disappear, and the maximum amplitude of the out-of-plane magnetic field is found in the center of the current sheet.

This work was supported by the Chinese Academy of Sciences (KJCX2YW-N28), the National Natural Science Foundation of China (40725013, 40974081 and 40931053), the Specialized Research Fund for State Key Laboratories and the Fundamental Researd Funds for the Central Universities (WK20800000010).

1 Biskamp D. Magnetic Reconnection in Plasmas. Cambridge: Cambridge University Press, 2000

2 Wang S, Lee L C. Magnetic Reconnection (in Chinese). Hefei: Anhui Education Press, 1999

3 Nishida A. Geomagnetic Diagnostics of the Magnetosphere. New York: Springer, 1978

4 Ge Y S, Russell C T. Polar survey of magnetic field in near tail: Reconnection rare inside $9 \mathrm{R}_{\mathrm{E}}$. Geophys Res Lett, 2006, 33: L02101

5 Wesson J. Tokomaks. New York: Oxford University Press, 1997

6 Sonnerup U O. Magnetic Field Reconnection, in Solar System Plasma Physics. In: Lanzerotti L T, Kennel C F, Parker E N, eds. Vol. 3. New York: North-Holland, 1979. 45-108

7 Birn J, Drake J F, Shay M A, et al. Geospace Environmental Modeling (GEM) magnetic reconnection challenge. J Geophys Res, 2001, 106: 3715-3719

8 Ma Z W, Bhattacharjee A. Hall magnetohydrodynamic reconnection: The Geospace Environment Modeling challenge. J Geophys Res, 2001, 106: 3773- 3782

9 Pritchett P L. Geospace Environment Modeling magnetic reconnection challenge: Simulations with a full particle electromagnetic code. J Geophys Res, 2001, 106: 3783-3798

10 Wang R S, Lu Q M, Huang C, et al. Multispacecraft observation of electron pitch angle distributions in magnetotail reconnection. J Geophys Res, 2010, 115: A01209

11 Lu Q M, Huang C, Xie J L, et al. Features of Separatrix regions in magnetic reconnection: Comparison of 2D particle-in-cell simulationns and Cluster observations. J Geophys Res, 2010, 115: A11208

12 Huang C, Wang R S, Lu Q M, et al. Electron density hole and quadruple structure of $\mathrm{B}_{\mathrm{y}}$ during collisionless magnetic reconnection. Chinese Sci Bull, 2010, 55: 718-722

13 Nagai T, Shinohara I, Fujimoto M, et al. Structure of the Hall current system in the vicinity of the magnetic reconnection site. J Geophys Res, 2003, 108: 1357

14 Øieroset M, Phan T D, Fujimoto M, et al. In situ detection of collisionless reconnection in the Earth's magnetotail. Nature, 2001, 412: $414-417$

15 Mozer F S, Bale S D, Phan T D. Evidence of diffusion regions at a subsolar magnetopause crossing. Phys Rev Lett, 2002, 89: 015002

16 Vaivads A, Khotyaintsev Y, Andre M, et al. Structure of the magnetic reconnection diffusion region from four-spacecraft observations. Phys Rev Lett, 2004, 93:105001

17 Fu X R, Lu Q M, Wang S. The process of electron acceleration during collisionless magnetic reconnection. Phys Plasmas, 2006, 13: 012309

18 Pritchett P L, Coroniti F V. Three-dimensional collisionless magnetic reconnection in the presence of a guide field. J Geophys Res, 2004, 109: A01220

19 Harris E G. On a plasma sheath separating regions of oppositely directed magnetic field. Nuovo Cimento, 1962, 23: 115

20 Huang C, Lu Q M, Wang S. The mechanisms of electron acceleration in antiparallel and guide field magnetic field. Phys Plasmas, 2010, 17: 072306

Open Access This article is distributed under the terms of the Creative Commons Attribution License which permits any use, distribution, and reproduction in any medium, provided the original author(s) and source are credited. 